\title{
Air fungal contamination in ten hospitals' food units from Lisbon
}

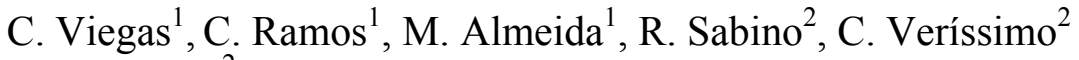 \\ \& L. Rosado ${ }^{2}$ \\ ${ }^{I}$ Higher School of Health Technologies of Lisbon, \\ Polytechnic Institute of Lisbon, Portugal \\ ${ }^{2}$ National Institute of Health Dr. Ricardo Jorge, Mycology Laboratory, \\ Portugal
}

\begin{abstract}
A descriptive study was developed to monitor air fungal contamination in ten food units from hospitals. Fifty air samples of 250 litres were collected through impaction method. Samples were collected in food storage facilities, kitchen, food plating, canteen and also, outside premises, since this is the place regarded as reference. Simultaneously, environmental parameters were also monitored, including temperature and relative humidity through the equipment Babouc, LSI Sistems and according to the International Standard ISO 7726. Thirty two species of fungi were identified in air, being the 2 genera most commonly found Penicillium sp. (43, 6\%) and Cladosporium sp. (23, 2\%). Regarding yeasts, only Rhodotorula sp. $(84,2 \%)$ and Trichosporon sp. $(15,8 \%)$ were isolated. There was coincidence between prevailing genera in interior and outside premises, however all ten food units presented fungal species different from the ones isolated from outside. Nine from the ten food units presented Aspergillus species, such as A. flavus, A. ochraceus, A. versicolor, A. candidus, A. fumigatus, A. niger and $A$. niveus. There was no significant relation $(\mathrm{p}>0,05)$ between fungal contamination, temperature and relative humidity.

Keywords: air, fungal contamination, food units, hospitals.
\end{abstract}

\section{Introduction}

Hospital-wide surveillance studies have shown the presence of various potentially pathogenic fungal species in health care settings [1]. The microbial 
population of a given environment is influenced by many factors including the number of visitors and the amount of materials brought in from outside. In some hospital areas, such as the operating theater and intensive care unit, the microorganism number are usually extremely low. This is due to the high sanitary standards as compared to other hospital areas [2] like food units. Fungi presence requires ideal conditions of temperature, humidity, oxygen, carbon sources, nitrogen and minerals. Their biological activities of biodegradation and biodeterioration, depend on their enzymes activity, the environmental conditions, the competition phenomenon and the nature of the substrate. In situations where the fungal concentrations are high or when people suffer from respiratory problems or have a weak immune system, exposure to fungi can cause the onset of symptoms and disease. The effects are dependent on the species present, the metabolic products, the concentration and exposure duration and individual susceptibility [3].

Fungal exposure in hospitals is of particular interest due to the possible patient's susceptibility. Therefore, it is important to contribute to the increase of knowledge referring air fungal contamination in hospitals indoor spaces, such as food units, in order to identify most effective preventive measures to avoid such contamination. This investigation was designed to describe environmental air fungal contamination in ten hospitals food units from Lisbon.

\section{Materials and methods}

A descriptive study was developed to monitor air fungal contamination in ten food units from hospitals during a six-month period. Fifty air samples of 250 litres were collected through impaction method. Samples were collected in food storage facilities, kitchen, food plating, canteen and also, outside premises, since this is the place regarded as reference. Simultaneously, environmental parameters were also monitored, including temperature and relative humidity through the equipment Babouc, LSI Sistems and according to the International Standard ISO 7726.

Air samples were collected at $140 \mathrm{~L} /$ minute and at one meter tall on to malt extract agar with antibiotic chloramphenicol (MEA), in the facilities, and also, outside premises, since this is the place regarded as reference.

After samples incubation, quantitative $\left(\mathrm{CFU} / \mathrm{m}^{3}\right)$ and qualitative results were obtained, with identification of isolated fungal species. Whenever possible, filamentous fungi were identified to the species level, since adverse health effects vary according to fungal species $[4,5]$. Identification of filamentous fungi was carried out on material mounted in lactophenol blue and achieved through morphological characteristics listed in illustrated literature [5] and yeasts were identified by using ID 32C strips (Marcy é Etoile, France) [6].

Tables with frequency distribution of isolated fungal species were made with the obtained data. Correlation between fungal concentration and temperature and relative humidity was also analyzed. 


\section{Results}

Thirty two species of fungi were identified in the collected samples from indoor air, being Penicillium and Cladosporium the two genera most commonly found, with $43,6 \%$ and $23,2 \%$ of frequency. Regarding yeasts, only Rhodotorula sp. $(84,2 \%)$ and Trichosporon sp. (15,8\%) were isolated (Table 1)

Table 1: $\quad$ Most frequent fungi identified in the food unit's air.

\begin{tabular}{|c|c|}
\hline Filamentous fungi & Frequency (\%) \\
\hline Penicillium sp. & 43,6 \\
Cladosporium sp. & 23,2 \\
Chrysonilia sp. & 7,3 \\
Chrysosporium sp. & 5,3 \\
Aspergillus sp. & 4,9 \\
Others & 15,7 \\
\hline Yeasts & Frequency (\%) \\
\hline Rhodotorula sp. & 84,2 \\
Trichosporon sp. & 15,8 \\
\hline
\end{tabular}

There was coincidence between prevailing genera in indoor and outside premises, however all ten food units presented fungal species different from the ones isolated from outside. Nine from the ten food units presented Aspergillus species, such as $A$. flavus, $A$. ochraceus, $A$. versicolor, $A$. candidus, A. fumigatus, $A$. niger and $A$. niveus.

Concerning comparison of concentrations found in air, for indoor and outdoor environments, nine of the ten food units showed higher levels of contamination in indoor air.

Concerning the influence of environmental variables monitored - temperature and relative humidity - no significant correlation $(p>0,05)$ was revealed.

\section{Discussion}

Concerning the predominant genus found in indoor air samples (Penicillium), there are different potential risks associated with their inhalation, due to the toxins release [7]. Regarding Cladosporium genus, is probably the fungus that occurs more frequently around world, especially in temperate climates [8] such as in Portugal. This genus is deeply connected to indoor condensation problems [9]. Both of the referred genus were also the more frequent in a study realized in a Portuguese poultry [10].

It is suggested that fungal levels found indoors should be compared, quantitatively and qualitatively, with those found outdoors, because the first are dependent on the last [3]. Nevertheless, when it comes to fungal levels, it should be taken into account that indoor and outdoor environments are quite different which, by itself, justifies diversity of species between different spaces. However, 
with regard to fungal contamination, there are no stipulated thresholds which makes essential to compare fungal levels indoors and outdoors.

Thus, indoor air quality that significantly differs from the outdoor could mean that there are infiltration problems and exist a potential risk for health. It is worth mentioning that as outdoor air is a major source of the fungi found indoors, it is no coincidence that, in this case, the prevailing genera, Penicillium sp. and Cladosporium sp., are the same in both these environments [11]. Nonetheless, all the monitored food units had one or more spaces with fungal species that differed from the ones isolated outside, and nine of them presented more $\mathrm{CFU} / \mathrm{m}^{3}$ in indoor than outdoor air, suggesting in both situations, fungal contamination from within [11].

With regard to qualitative assessment of fungal contamination in air, it is suggested that, among other species, Aspergillus fumigatus and Penicillium, Trichoderma, Fusarium and Ulocladium species, all of them isolated in the present study, are regarded as indicators of humidity problems or potential risk to health [3]. Moreover, according to American Industrial Hygiene Association (AIHA), in 1996, for determination of biological contamination in environmental samples, confirmed presence of the species Aspergillus flavus and Aspergillus fumigatus, both identified in this study, requires implementation of corrective measures [12].

Taking into account what is mentioned in Portuguese law, that $500 \mathrm{CFU} / \mathrm{m}^{3}$ is the maximum reference concentration in indoor air, was exceeded in four indoor spaces analyzed in this study. Regarding what is mentioned in Portuguese Technician Norm NT-SCE-02, the presence of opportunistic fungi from Aspergillus genus, shows a lack of air quality in indoor space. Moreover, Aspergillus species are frequently present on food and thus can be an indirect source of airway or digestive tract colonization of the patients [13].

Results related to environmental variables are not consistent with what is expected [14]. It was found that the relationship between the fungal air contamination and the temperature and relative humidity was not statistically significant $(p>0,05)$. This may be justified by the effect of other environmental variables also influencing fungal spreading, namely workers and food, who may carry a great diversity of fungal species [15], as well the developed activities that may also affect fungal concentration [16].

\section{Conclusions}

With this study, was possible to observe that food units can be a source of fungal contamination to other hospital ward as well as for patients' food.

Unlike other studies, the monitored environmental variables (temperature and relative humidity) did not show the expected association with fungal concentration, which may possibly have resulted from other variables not investigated in this study. 


\section{References}

[1] Sarica S, Asan A, Otkun M \& Ture M., Monitoring indoor airborne fungi and bacteria in the different areas of Trakya University Hospital, Edirne, Turkey. Indoor Built Environment, 11, pp. 285 - 292, 2002.

[2] Ekhaise F, Ighosewe O \& Ajakpovi O., Hospital indoor airborne microflora in private and government owned hospitals in Benin city, Nigeria. Word Journal of Medical Sciences, 3 (1), pp. 19 - 23, 2008.

[3] Goyer N, Lavoie J, Lazure L \& Marchand G., Bioaerosols in the Workplace: Evaluation, Control and Prevention Guide. Institut de Recherche en Santé et en Sécurité du Travail du Québec, 2001.

[4] Rao C, Burge H \& Chang J., Review of quantitative standards and guidelines for fungi in indoor air. J Air Waste Manage Assoc., 46, pp. $899-908,1996$.

[5] Hoog C, Guarro J, Gené G \& Figueiras M., (2 $2^{\text {th }}$ ed). Atlas of Clinical Fungi. Centraalbureau voor Schimmelcultures, 2000.

[6] Ghannoum M, Hajeh R, Scher R, Konnikov N, et al., A large-scale North American study of fungal isolates from nails: The frequency of onychomycosis, fungal distribution and antifungal susceptibility patterns. J. Am. Acad. Dermatol, 43, pp. 641 - 648, 2000.

[7] Kemp P, Neumeister-Kemp H, Esposito B, Lysek G \& Murray F., Changes in airborne fungi from the outdoors to indoor air; Large HVAC systems in nonproblem buildings in two different climates. American Industrial Hygiene Association, 64, pp. 269 - 275, 2003.

[8] Cooley J, Wong W, Jumper C \& Straus D., Correlation between the prevalence of certain fungi and sick building syndrome. Occup. Environ Med, 55, pp. $579-584,1998$.

[9] Garret M, Rayment P, Hooper M, Abranson M \& Hooper B., Indoor Airborne Fungal Spores, House Dampness and Associations with Environmental Factors and Respiratory Health in Children. Clinical and Experimental Allergy, 28, pp. 459 - 467, 1998.

[10] Viegas C, Verissimo C, Rosado L \& Silva Santos C., Poultry fungal contamination as a public health problem. Environmental Toxicology III. WIT Transactions on Biomedicine and Health, 2010.

[11] Nevalainen A., Bio-aerosols as exposure agents in indoor environment in relation to asthma and allergy. Section 3 Asthma and allergy. Proceedings of the First ENVIE Conference on Indoor Air Quality and Health for EU Policy, Helsinki, Finland, 2007.

[12] American Industrial Hygiene Association: Field Guide for the Determination of Biological Contaminants in Environmental Samples. AIHA, 1996.

[13] Sarfati J, Jensen H \& Latgé J., Route of infections in bovine aspergillosis. J Med Vet Mycol, 34, pp. 379 - 383, 1996.

[14] Kakde U, Kakde H \& Saoji A., Seasonal Variation of Fungal Propagules in a Fruit Market Environment, Nagpur (India). Aerobiologia, 17, pp. $177-182,2001$. 
132 Food and Environment

[15] Scheff P, Pulius V, Curtis L \& Conroy L., Indoor air quality in a middle school, Part II: Development of emission factors for particulate matter and bioaerosols. Applied Occupational and Environmental Hygiene, 15, pp. $835-842,2000$.

[16] Buttner M \& Stetzenbach L., Monitoring Airborne fungal spores in an experimental indoor environment to evaluate sampling methods and the effects of human activity on air sampling. Applied and Environmental Microbiology, 59, pp. 219-226, 1993. 